\title{
SURFACE PROPERTIES OF T TAURI STARS
}

\author{
CLAUDE BERTOUT AND CATHERINE MENNESSIER \\ Observatoire de Grenoble \\ Université Joseph Fourier \\ F-38041 Grenoble Cedex 9 \\ France
}

\begin{abstract}
We focus here on those $\mathrm{T}$ Tauri star properties that make it worthwhile to Doppler-image these young stellar objects, namely magnetic structures and accretion features. We then comment on recent results obtained for two bright, fast rotating weak-line $\mathrm{T}$ Tauri stars.
\end{abstract}

\section{Introduction}

$\mathrm{T}$ Tauri stars are late-type stars located in star-forming regions such as the Taurus-Auriga or the $\rho$-Ophiuchi molecular clouds. Main arguments for their youth include (i) kinematic association with these clouds and (ii) high abundance of lithium in their atmospheres, which signals the pre-main sequence state of evolution of these objects. In the Hertzsprung-Russell diagram, low-mass T Tauri stars are located on the Hayashi track corresponding to their mass and the most massive are approaching the main sequence on radiative tracks. For a full description of $\mathrm{T}$ Tauri star observational properties, the interested reader is referred to Cohen and Kuhi (1979) and Appenzeller and Mundt (1989). The most striking property of these objects is their light variability on all time-scales and at all wavelengths. While a wide range of models were proposed over the years to explain one aspect or the other of T Tauri activity, the current, consensual paradigm postulates that a cold circumstellar disk surrounds, and interacts with, a late-type magnetically active star. Arguments for and against this model are discussed, e.g., by Bertout (1989). According to this model, thus, there are two broad physical phenomena that play a role in $T$ Tauri stars: magnetic activity and matter accretion. 
Magnetic activity is most easily studied in the so-called weak-emission line T Tauri stars (WTTSs). This class of young stellar objects was recently discovered through strong X-ray (and sometimes radio) activity. WTTSs display little optical activity except for modest light variations (cf. Feigelson, Giampapa and Vrba 1991). They are moderately fast rotators with typical equatorial velocities in the range $10-30 \mathrm{~km} / \mathrm{s}$ and their magnetic activity appears commensurate with their rotation rates.

Accretion is the trademark of the classical T Tauri stars (CTTSs), which were discovered in the course of Ha surveys. They rotate a bit more slowly than WTTSs (Bouvier et al. 1993) and also display less magnetic activity (Gahm 1994). Observational evidence for matter accretion includes strong IR and UV continuum excesses that can be modeled by accretion disks (e.g., Basri and Bertout 1989) and high-velocity $(200-400 \mathrm{~km} / \mathrm{s})$ red-displaced absorption components in the Balmer emission lines (Edwards et al. 1994).

We summarize in the next section observational evidence for inhomogeneous $\mathrm{T}$ Tauri surface brightness which can result from both stellar magnetic activity and accretion. We then discuss expected properties of accretion regions on the stellar surface. Finally, we review current Doppler imaging results for WTTSs and discuss future prospects.

\section{Photometric evidence for complex surfaces}

While quasi-periodic light variations on time-scales of a few days were reported for CTTSs already by Herbig (1962), truly periodic variability was first noted for some WTTSs such as V410 Tau (Schaefer 1983, Rydgren and Vrba 1983, Vrba, Herbst and Booth 1988) and interpreted as rotational modulation due to inhomogeneous temperature distributions. Crude spot models were constructed to account for the variability of both WTTSs and CTTSs (e.g., Bouvier, Bertout and Bouchet 1986, Bouvier and Bertout 1989, Herbst 1989, Bouvier et al. 1993).

The photometric light-curves of WTTSs are characterized by approximately constant amplitudes (typically 0.05 to $0.5 \mathrm{mag}$ ) and stable periods over several years. The surface spots responsible for variability are thus long-lived and cover a large fraction of the surface. The wavelength dependence of the light curves indicates that the large spots responsible for WTTSs variability are colder than the overall stellar photosphere. Spot properties derived from simple models are strikingly similar to those of spots found on magnetically active late-type stars with similar rotation rates (Bouvier and Bertout 1989). The relevance of this comparison is also supported by the average magnetic field strength of $1 \mathrm{kG}$ over the entire stellar surface found for the bright WTTS TAP35 (Basri, Marcy and Valenti 1992). 
CTTSs exhibit a much more complex behavior than WTTSs. Large cold spots similar in nature to the magnetic spots of WTTSs are clearly present on many of them. Some of them display occasional evidence for the presence of localized surface regions with temperature hotter than the photosphere (e.g., DF Tau; cf. Bertout, Basri and Bouvier 1988). Phases of periodic and aperiodic variability alternate, and the photometric period of stars showing evidence for hot regions on their surfaces can vary by as much as $30 \%$ in a year or so (Bouvier and al. 1995). The reason for these period variations remains unknown.

\section{Surface properties due to accretion}

In spite of fairly direct evidence for matter accretion onto the surface of $\mathrm{T}$ Tauri stars, its physics remains elusive. Two simplified models were investigated: accretion through a boundary layer (e.g., Lynden-Bell and Pringle 1974, Regev and Bertout 1995, Godon, Regev and Shaviv 1995) and magnetically-controlled accretion along stellar magnetic field lines (Bertout, Basri and Bouvier 1988, Königl 1991).

\subsection{BOUNDARY LAYER ACCRETION}

In the classical accretion disk boundary layer (BL), accretion is axisymmetric and occurs at subsonic speed, giving rise to a hot equatorial belt (Popham et al. 1993, Regev and Bertout 1995). Emitted BL radiation is compatible with UV and optical excess radiation observed in CTTSs. However, the assumption of axisymmetry is a serious flaw for a disk that is probably the site of various non-axisymmetric instabilities. Observations of hot spots in fact contradict this assumption. In addition, the BL model has problems with two observational findings. First, inverse P Cygni absorption components at the Balmer lines indicate supersonic infall. Second, accretion of disk matter with high specific angular momentum should accelerate the stellar rotation, in contrast to observations.

We note, however, that these two arguments against the BL model are not as strong as they seem at first. On one hand, even though subsonic accretion is expected in steady-state disk models (Narayan, Loeb and Kumar 1994), it can occur supersonically in non-steady boundary layers when the kinematic viscosity is large (Godon 1996). On the other hand, the rotational velocity balance of the star depends crucially on the (unknown) mechanism for driving the observed mass ejection. It is our opinion, therefore, that BL accretion cannot be entirely ruled out at this point, particularly in the view that it provides an emitting zone with strong velocity gradients where optical depth effects might possibly account for the apparent changes in period detected in accreting stars. 


\subsection{MAGNETICALLY CONTROLLED ACCRETION}

This model assumes that the inner part of the disk is disrupted by a strong stellar magnetic field, so that accretion occurs primarily along the magnetic field lines. If the value of the field strength found for TAP35 is also characteristic of CTTS fields, it is obvious that the stellar field must have some influence on the accretion process. Königl (1991) considered accretion properties resulting from a stellar dipole field. If magnetic axis and rotation axis are aligned then high latitude accretion belts are formed; otherwise, accretion spots follow.

The main advantage of this model is to account easily for supersonic accretion observed in CTTSs and even more prominently in a CTTS subclass called YY Orionis stars (Walker 1972). However, detailed models of the spectrum emitted by an accretion flow of this type are not available, so that direct comparison with observations is not possible. The model is nevertheless popular because magnetic coupling between star and disk can account for the rotation rates of CTTSs (Cameron and Campbell 1993). We mentioned earlier, however, that the crucial role of winds in carrying away angular momentum is not considered in this model. Also, current models for both magnetically-controlled matter accretion (e.g., Königl 1991) and generation of the powerful $\mathrm{T}$ Tauri winds at the interface between stellar magnetosphere and disk (Shu et al. 1994) assume a dipole field geometry, whereas the magnetic field in CTTSs is likely to be less organized, resulting in the patchy surface temperature distribution inferred by Herbig and Soderblom (1980) from observations of the calcium infrared triplet.

In conclusion, while stellar magnetic fields are likely to play a role in the disk/star interaction, it is not entirely clear that the field geometry is as simple as envisioned in current models. A more realistic picture might be a "dirty accretion" model where numerous magnetic loops interact with the disk without dynamically disrupting it on large scales. In this admittedly speculative picture, a (non-steady) magnetic boundary layer forms at the stellar equator and radiates most of the accretion energy while some accretion occurs also along field lines and is responsible for the weak reddisplaced absorption components seen in CTTSs.

In view of the current uncertainties concerning both the magnetic field topology and the geometry of accretion onto T Tauri surfaces, it should be obvious at this point that Doppler imaging promises to be a very helpful investigation tool.

\section{First results in Doppler imaging of $T$ Tauri stars}

There are major difficulties involved in imaging $\mathrm{T}$ Tauri stars, and particularly the CTTSs. The first one relates to their location in molecular clouds; 
the closest star-forming regions are at distances $120-150 \mathrm{pc}$, and T Tauri stars are typically obscured by $1 \mathrm{mag}$ in the visual because of the presence of circumstellar and intracloud dust. A typical CTTS is a K7V star with $\mathrm{V}=13 \mathrm{mag}$. Furthermore, the spectrum of CTTSs is disturbed by "veiling", an excess blue and ultraviolet continuum attributed to the star/disk interaction region that fills up the photospheric absorption lines and affects their equivalent widths. Veiling must be determined precisely before comparing data to computed spectral lines. Also, classical line formation codes are probably not entirely relevant to the complex atmospheres of $T$ Tauri stars. For all these reasons, Doppler imaging of CTTSs such as DF Tau or BP Tau, which can be seen as the prototypes of the class, remains a formidable challenge. First attempts were presented in this meeting (posters by Unruh et al. and Petrov et al.).

Luckily, while less exotic, the WTTSs are quite interesting on their own right and some of them, albeit not the most typical, are relatively bright. So far, Doppler images were computed for V410 Tau (Strassmeier, Welty and Rice 1994, Joncour, Bertout and Menard 1994, and Hatzes 1995), and for HDE 283572 (Joncour, Bertout and Bouvier 1994). We summarize these results below.

\subsection{V410 TAU}

One of the best studied WTTSs, V410 Tau was variously classified as a $\mathrm{K} 7, \mathrm{~K} 4$ or K2 star. The latter value was determined by Basri and Batalha (1990) on the basis of Hamilton spectrograms, and is probably the most accurate type. The star shows various manifestations of magnetic activity ranging from strong X-ray flux to radio flares (Cohen and Bieging 1986) and its period is well determined (Petrov et al. 1994). Its $\mathrm{V}$ magnitude varies from 10.7 to 11.1 , which indicates a substantial spot coverage.

All Doppler images agree on the presence of a large cold spot at high latitude that does not, however, cover the pole. In addition, there are less extended structures at intermediate latitude, and Strassmeier, Welty and Rice (1994) find evidence for localized hot spots that could not have been detected by the other groups because of the inversion techniques they used. If confirmed by future studies, the presence of hot spots would be quite puzzling since V410 Tau is not believed to be surrounded by an accretion disk. The fact that everyone agrees on the location and approximate extent of the high-latitude structure is quite encouraging since the observations and data inversion techniques used by the various groups are quite different. As noted by Hatzes (1995), high-latitude magnetic activity is supported by the radio flux modulation, which can be interpreted in the framework of a large magnetic loop extending above the stellar pole (Bieging and Cohen 
1989). In any case, there is no evidence for a large-scale dipole field in this star. The persistence of the spot structure over the time interval 1990-1994 is reminiscent of the RS CVn case.

\subsection{HDE 283572}

This WTTS was discovered by Walter et al. (1987) during an X-ray survey of star-forming regions. It is a 9th magnitude G5IV star which displays strong magnetic activity. The Doppler image derived by Joncour, Bertout and Bouvier (1994) shows an extended, cold polar cap. It is striking that this fast-rotating WTTS (its projected rotational velocity is about $90 \mathrm{~km} / \mathrm{s}$ ) and fast-rotating RS CVn stars display similar polar spots in spite of their different evolutionary status. This result supports the model of spot appearance proposed by Schüssler and Solanki (1992). Note however that the Doppler image of the bright CTTS SU Aur presented by Petrov et al. in a poster at this meeting displays two large equatorial dark spots, although the equatorial velocities of SU Aur and HDE 283572 are similar. It is thus tantalizing to speculate that the accretion disk of SU Aur might play a role in inhibiting the formation of polar spots.

\section{Conclusions and outlook}

Doppler imaging is obviously an extremely promising technique for studying T Tauri stars. So far, only the brightest objects can be studied and results amply demonstrate the potentialities of the technique for looking at the signatures of magnetic fields and accretion on the surfaces of $\mathrm{T}$ Tauri stars. In order to image the most typical, fainter young stellar objects, the luckiest among us will be able to obtain extremely high-quality data at major facilities such as the Keck telescope. Less fortunate astronomers will try to find clever ways to improve the inversion algorithms. This is the path that we are following, by necessity more than by virtue.

The exploratory code that was developed for the studies of Joncour, Bertout and Menard (1994) and Joncour, Bertout and Bouvier (1994) was used in conjunction with the data of limited $\mathrm{S} / \mathrm{N}$ and resolution that can be obtained with $2 \mathrm{~m}$-class telescopes on the brightest $\mathrm{T}$ Tauri stars. Typically, the data we collect on these telescopes have $S / N \approx 100$ and $R \approx 30000$, so that we can only detect brightness inhomogeneities larger than about 10smaller structures. In other words, we cannot expect to detect hot accretion spots, with typical area 1approach. In recent months, we thus embarked in several studies aimed at quantitatively assessing the relationship between data quality and stability of the reconstruction process. Among the several ideas that we are testing, we try to use regularization criteria that take into account the resolution variation as a function of spot location on the stellar 
disk. We feel that such criteria are potentially more relevant to the problem at hand than entropy maximization. Also, we are trying to model the data noise, using sophisticated signal processing techniques. Finally, we explore the usefulness of neural network techniques for recognizing line profile patterns, in order to infer an approximate image of the star that can be used as a starting point in the reconstruction process. We evidently hope that these efforts will allow us in a not too distant future to Doppler-image typical CTTSs from spectroscopic data obtained on 4-meter class telescopes.

\section{References}

Appenzeller, I. and Mundt, R. (1989), A\&AR 1, 291.

Basri, G. and Batalha, C. (1990), ApJ 363, 654.

Basri, G. and Bertout, C. (1989), ApJ 341, 340.

Basri, G. S., Marcy, G. W. and Valenti, J. A. (1992), ApJ 390, 622.

Bertout, C. (1989), ARA\&A 27, 351.

Bertout, C., Basri, G. and Bouvier, J. (1988), ApJ 330, 350.

Bieging, J. H. and Cohen, M. (1989), AJ 98, 1686.

Bouvier, J. et al. (1995), A\&A, in press.

Bouvier, J. and Bertout, C. (1989), A\&A 211, 99.

Bouvier, J., Bertout, C. and Bouchet, P. (1986), A\&A 158, 149.

Bouvier, J. et al. (1993), A\&A 272, 176.

Cameron, A. C. and Campbell, C. G. (1993), A\&A 274, 309.

Cohen, M. and Bieging, J. H. (1986), AJ 92, 1396.

Cohen, M. and Kuhi, L. V. (1979), ApJS 41, 743.

Edwards, S. et al. (1994), AJ 108, 1056.

Feigelson, E. D., Giampapa, M. S. and Vrba, F. J. (1991), in The Sun in Time, eds. G.

R. Sonnett, M. S. Giampapa and M. S. Matthews. Tucson: Univ. of Arizona Press.

Gahm, G. F. (1994), in IAU Coll. 151, Flares and Flashes, Sonneberg.

Godon, P. (1996), ApJ, in press.

Godon, P., Regev, O. and Shaviv, G. (1995), MNRAS 275, 1093.

Hatzes, A. P. (1995), ApJ 451, 784.

Herbig, G. H. (1962), Advances A\&A 1, 47.

Herbig, G. H. and Soderblom, D. R. (1980), ApJ 242, 628.

Herbst, W. (1989), AJ 98, 2268.

Joncour, I., Bertout, C. and Bouvier, J. (1994), A\&A 291, L19.

Joncour, I., Bertout, C. and Menard, F. (1994), A\&A 285, L25.

Königl, A. (1991), ApJ 370, L39.

Lynden-Bell, D. and Pringle, J. E. (1974), MNRAS 168, 603.

Narayan, R., Loeb, A. and Kumar, P. (1994), ApJ 431, 359.

Petrov, P. P. et al. (1994), A\&AS 107, 9.

Popham, R. et al. (1993), ApJ 415, L127.

Regev, O. and Bertout, C. (1995), MNRAS, in press.

Rydgren, A. E. and Vrba, F. J. (1983), ApJ 267, 191.

Schaefer, B. (1983), ApJ 266, L45.

Schüssler, M. and Solanki, S. K. (1992), A\&A 264, L13.

Shu, F. et al. (1994), ApJ 429, 781.

Strassmeier, K. G., Welty, A. D. and Rice, J. B. (1994), A\&A 285, L17.

Vrba, F. J., Herbst, W. and Booth, J. F. (1988), AJ 96, 1032.

Walker, M. F. (1972), ApJ 175, 89.

Walter, F. M. et al. (1987), ApJ 314, 297. 


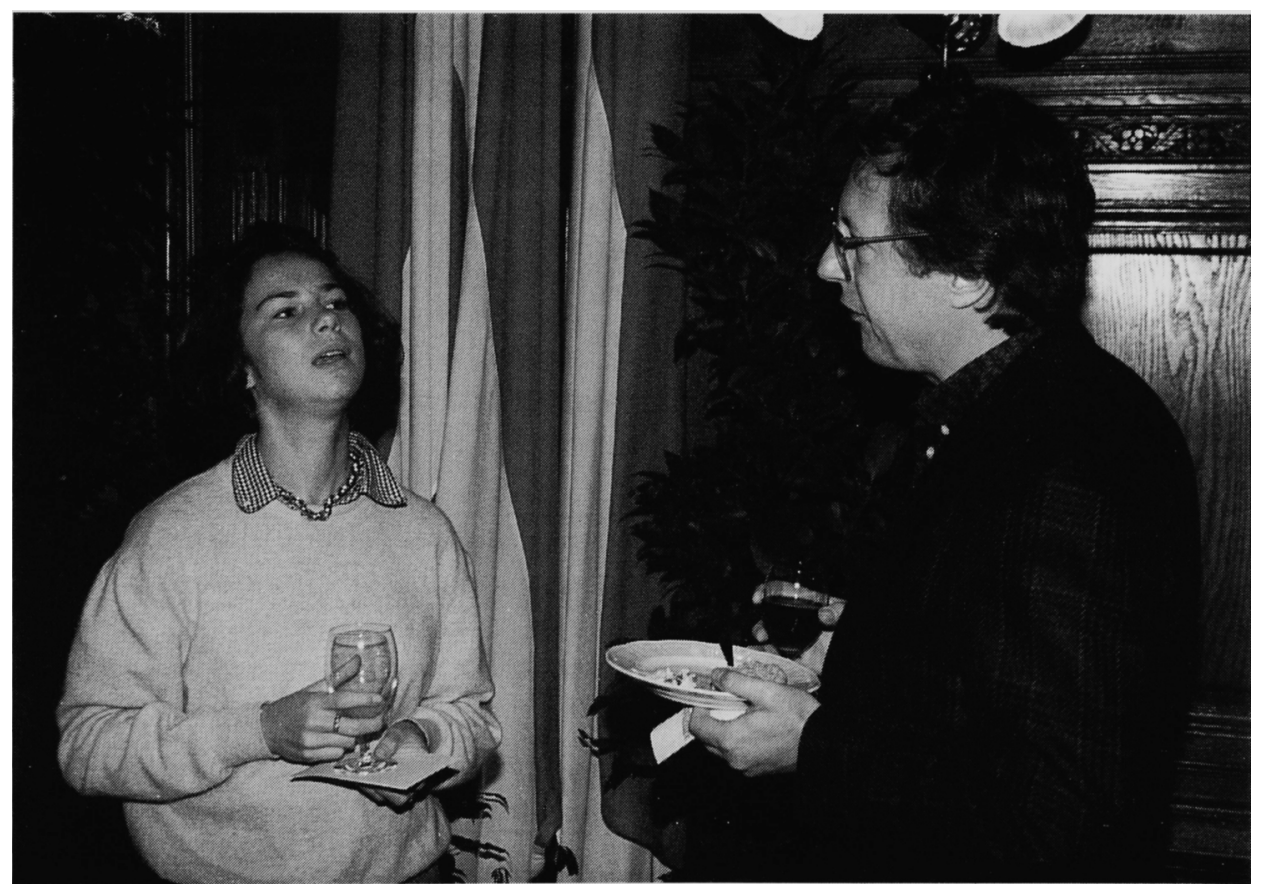

Catherine Mennessier (left) arguing with her thesis adviser Claude Bertout. Good luck.

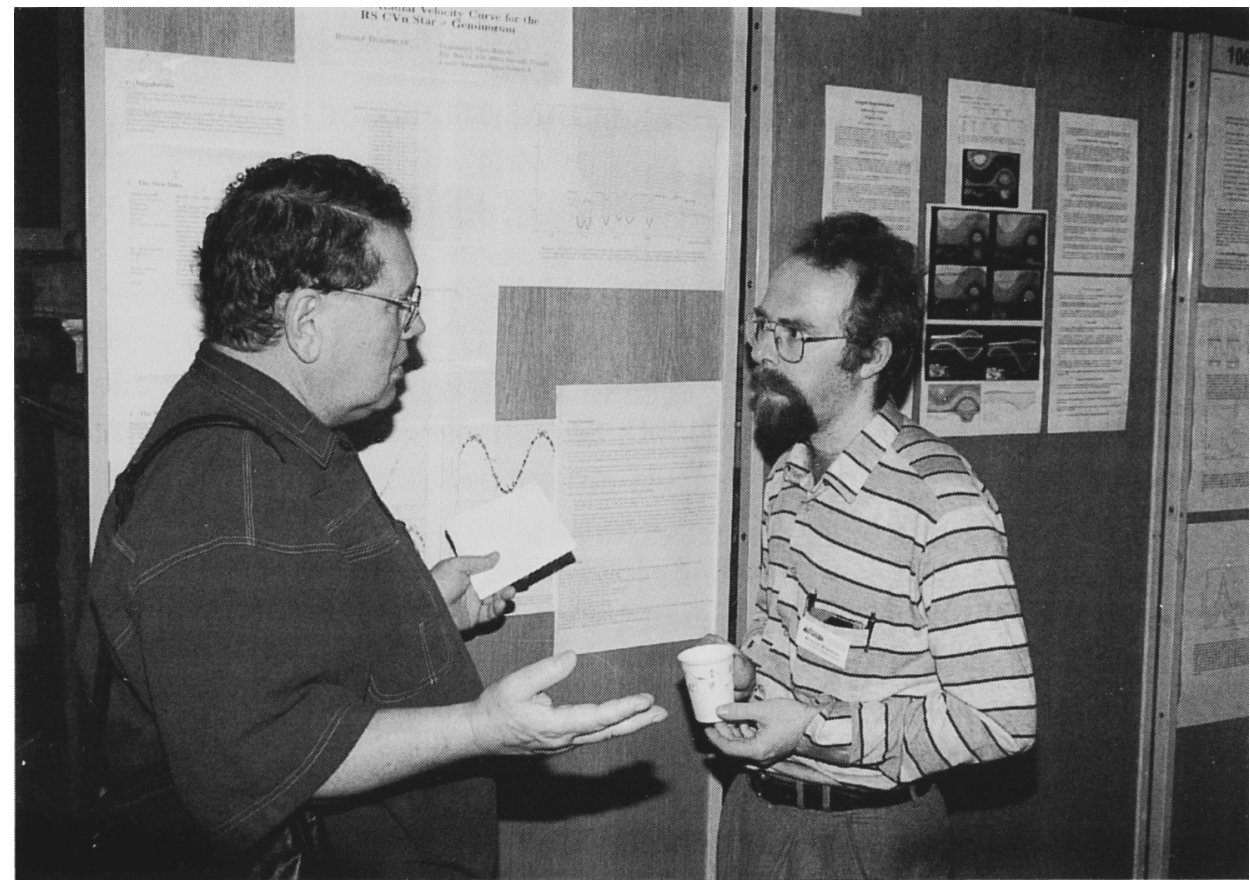

It's poster time: Øystein Elgarøy (left) and Rudolph Dümmler on $\sigma$ Gem. 